\title{
Uso de Álcool e outras Substâncias Psicoativas por Estudantes Universitários de Psicologia ${ }^{1}$
}

\author{
Isabella Tereza Martins Pires ${ }^{1}$ \\ ${ }^{1}$ Universidade Federal de Uberlândia, MG, Brasil. \\ Marciana Gonçalves Farinha ${ }^{1}$ \\ ${ }^{1}$ Universidade Federal de Uberlândia, MG, Brasil.
}

\author{
Sandra Cristina Pillon ${ }^{2}$ \\ ${ }^{2}$ Universidade de São Paulo, SP, Brasil. \\ Manoel Antônio dos Santos ${ }^{2}$ \\ ${ }^{2}$ Universidade de São Paulo, SP, Brasil.
}

Resumo: O consumo de substâncias psicoativas (SPAs) cresce em escala global, especialmente entre universitários. Este estudo tem como objetivo avaliar o padrão de uso de álcool e outras SPAs em estudantes universitários. A amostra foi composta por 180 graduandos de Psicologia que preencheram um questionário sociodemográfico e instrumentos de rastreamento do uso de SPAs validados para o contexto brasileiro. Os resultados evidenciaram maior prevalência do uso de álcool: $81,7 \%$ na vida, $67,6 \%$ nos últimos três meses e $55 \%$ no padrão binge. Dentre os motivos endossados para o consumo destaca-se o convívio universitário, porém o fator mais valorizado foi o álcool como potencializador do desejo de fumar, seguido de sua percepção como fator gregário em festas/ reuniões sociais e como facilitador do enfrentamento de estresse. As pressões da vida universitária geram insegurança e instabilidade que deixam os jovens universitários mais suscetíveis à influência dos pares e à busca de diversão e descontração em ambientes de festas, nas quais o consumo é estimulado e facilitado, o que pode favorecer o beber em quantidade excessiva. Esse padrão de consumo pode se associar a outros comportamentos de risco, como dirigir sob efeito do álcool, manter relações sexuais sem proteção ou envolver-se em distúrbios da ordem pública e infrações à lei. Considerando que os jovens fazem uso em excesso de SPAs sem um suporte social comunitário adequado, é necessário investir em programas de prevenção e políticas públicas guiadas pelas noções de autocuidado, protagonismo e participação ativa na própria reabilitação.

Palavras-chave: Droga (uso), Estudantes, Psicologia, Saúde Mental, Prevenção do Abuso de Drogas.

\section{Use of Alcohol and other Psychoactive Substances by Psychology Students}

\begin{abstract}
The consumption of psychoactive substances (SPAs) grows worldwide, especially among university students. This study evaluated the pattern of alcohol use and other SPAs in psychology students. The sample consisted of 180 Psychology undergraduates who filled out a sociodemographic questionnaire and instruments for tracking the use of SPAs validated for the Brazilian context. The results showed a higher prevalence of alcohol use: $81.7 \%$ in life, $67.6 \%$ in the last three months and 55\% following a binge pattern. Among the reasons endorsed for the consumption stand out university conviviality, but the most valued factor was alcohol as a potentiator of the desire to smoke, followed by its perception as a gregarious factor in parties/ social gatherings and as a facilitator to cope with stress situations. The pressures of university life create insecurity and instability, which make university students more susceptible to peer influence and the search for fun and relaxation in party environments where drinking is stimulated and facilitated, which may favor binge drinking. This pattern of consumption
\end{abstract}

\footnotetext{
${ }^{1}$ Apoio Fundação de Amparo à Pesquisa do Estado de Minas Gerais (FAPEMIG).
} 
may be associated to other risk behaviors, such as driving under the influence of alcohol, having unprotected sex, or engaging in public order disturbances and infractions of the law. Considering that these young people make excessive use of SPAs without adequate social and community support, it is necessary to invest in prevention programs and public policies guided by the notions of self-care, protagonism and active participation in self rehabilitation.

Keywords: Drug Usage, College Students, Psychology, Mental Health, Drug Abuse Prevention.

\title{
Uso de Alcohol y otras Sustancias Psicoactivas por Estudiantes Universitarios de Psicología
}

\begin{abstract}
Resumen: El consumo de sustancias psicoactivas (SPA) crece a escala global, especialmente entre universitarios. Este estudio tuvo como objetivo evaluar el patrón de uso de alcohol y otras SPA por estudiantes universitarios. Compusieron la muestra 180 estudiantes de graduación en Psicología, que respondieron a un cuestionario sociodemográfico e instrumentos de rastreo del uso de SPA validados para el contexto nacional. Los resultados evidenciaron mayor prevalencia del uso de alcohol: el $81,7 \%$ en la vida, el $67,6 \%$ en los últimos tres meses y el $55 \%$ en el patrón binge. Entre los motivos endosados para el consumo se destaca la convivencia universitaria, pero el factor más valorado fue el alcohol como potenciador del deseo de fumar, seguido de su percepción como factor gregario en fiestas/reuniones sociales y como facilitador del enfrentamiento de situaciones de estrés. Las presiones de la vida universitaria generan inseguridad e inestabilidad que dejan a los jóvenes universitarios más susceptibles a la influencia de los pares y a la búsqueda de diversión y relajación en fiestas y discotecas donde se estimula y facilita el consumo de alcohol, lo que puede favorecer la costumbre de beber en cantidad excesiva. Ese patrón de consumo puede asociarse a otros comportamientos de riesgo, como conducir bajo el efecto del alcohol, mantener relaciones sexuales sin protección o involucrarse en disturbios del orden público e infracciones a la ley. Considerando que estos jóvenes hacen uso excesivo de SPA sin un soporte social-comunitario adecuado, son necesarios programas de prevención y políticas públicas guiadas por las nociones de autocuidado, protagonismo y participación activa en la propia rehabilitación.
\end{abstract}

Palabras clave: Consumo de Drogas (Uso), Estudiantes Universitarios, Psicología, Saúde Mental, Prevención en el Abuso de Drogas.

O consumo de substâncias psicoativas (SPAs) entre jovens é um fenômeno que tem despertado crescente preocupação e desencadeado inúmeros problemas familiares, sociais e legais, além de complicações físicas e psíquicas para os usuários (Silva et al., 2016; United Nations Office on Drugs and Crime, 2017; Vilela, Silva, Grandi, Rocha, \& Figlie, 2016). O uso abusivo está alcançando rapidamente estudantes dos mais variados níveis educacionais e socioeconômicos (Andrade, Duarte, \& Oliveira, 2010; Soares, Barros, Araújo, Finelli, \& Jones, 2014). O presente estudo tem como foco o uso abusivo dessas substâncias no contexto acadêmico, além de buscar contribuir para a compreensão e planejamento de ações e políticas de prevenção relacionadas às SPAs.

As SPAs são substâncias capazes de alterar o nível de consciência, humor, comportamento e cognição de um indivíduo. O consumo está presente em todas as culturas e não se limita a determinados grupos étnicos, faixa etária, cor, gênero ou orientação sexual, embora existam diferenças dentro de cada um desses marcadores (Santos, Pereira, \& Siqueira, 2013). O uso de SPAs, principalmente o álcool, está associado de forma direta ou indireta à busca de fruição do prazer, beleza, popularidade e sucesso financeiro, cujos padrões são difundidos socialmente e veiculados especialmente 
pelos meios de comunicação de massa, que são considerados um fator de risco relevante para a propagação dos hábitos de consumo na sociedade contemporânea. As pessoas que convivem no ambiente universitário tendem a associar o consumo de álcool e tabaco à exposição prolongada e permissiva a essas drogas (Pedrosa, Camacho, Passos, \& Oliveira, 2011).

$\mathrm{O}$ ingresso na universidade, além de representar um marco de conquista na trajetória da maioria dos(as) jovens que conseguem ultrapassar a barreira do vestibular, também pode se tornar um período crítico de transição psicossocial, pois o(a) estudante se torna mais independente da supervisão dos pais, assume algumas responsabilidades e toma decisões a respeito de sua própria vida, o que em alguns casos pode produzir insegurança e instabilidade, deixando os(as) universitários(as) mais vulneráveis para o início do uso de substâncias (Santos, Pereira, \& Siqueira, 2013). Estudos epidemiológicos sobre o consumo de álcool e/ou outras drogas entre jovens no mundo e no Brasil apontam que o uso tem sido cada vez mais precoce, não se limitando somente à vida universitária, mas instalando hábitos que se iniciam na passagem da infância para a adolescência.

Pesquisa realizada nos Estados Unidos estimou que cerca de três milhões de crianças e adolescentes fumam tabaco e ingerem álcool pelo menos uma vez por mês, representando mais de $50 \%$ dos alunos de séries equivalentes ao ensino médio no Brasil (Marques \& Cruz, 2000). No cenário brasileiro, foi realizado o Segundo Levantamento Nacional de Álcool e Drogas (II Lenad) em 2012 (Laranjeiras, Madruga, Pinsky, Caetano, \& Mitsuhiro, 2012). Os dados apontaram que a população que experimenta álcool antes da vida adulta aumentou significativamente no período entre 2006 e 2012. Entre brasileiros(as) adultos(as), em 2006 13\% haviam ingerido bebidas alcoólicas com menos de 15 anos, enquanto $22 \%$ declararam ter experimentado com menos de 15 anos em 2012.

O álcool é de longe a droga de uso recreativo mais utilizada em contexto universitário, segundo estudo patrocinado pela Secretaria Nacional de Políticas sobre Drogas (Andrade et al., 2010). O primeiro Levantamento Nacional Sobre Uso de Álcool, Tabaco e Outras Drogas entre Universitários foi realizado em 27 capitais brasileiras. Os resultados revelaram que aproximadamente $80 \%$ dos(as) jovens adultos(as) de universidades públicas e particulares já fizeram uso de algum tipo de bebida alcoólica e $47 \%$ já usaram tabaco pelo menos uma vez na vida. Considerando os últimos doze meses, as substâncias utilizadas mais frequentemente foram o álcool (72\%) e o tabaco (27,8\%).

$\mathrm{O}$ binge-drinking é caracterizado pelo beber em quantidade excessiva em um curto período de tempo (National Institute on Alcohol Abuse and Alcoholism [NIAAA], 2016). Esse conceito diz respeito ao consumo de cinco ou mais doses consecutivas pelo menos uma vez nas últimas duas semanas, no caso dos homens, ou quatro ou mais doses no mesmo período, no caso das mulheres. Esse padrão de consumo é conhecido como um comportamento que incrementa os danos relacionados ao álcool na vida do indivíduo que faz uso diário dessa substância (Arantes, 2012).

Um dos aspectos a se destacar é que o álcool, a droga mais utilizada pela população mundial e considerada lícita na maioria dos países, frequentemente acarreta graves consequências físicas, psíquicas e sociais ao indivíduo que o consome em quantidade demasiada. A facilidade com que essa droga é encontrada e comercializada tem favorecido seu alto consumo e despertado diversos questionamentos acerca do uso abusivo, gerando reflexões a respeito da efetividade de possíveis medidas de prevenção e estratégias para a redução do consumo (Gomes \& Capponi, 2011; Nimtz et al., 2014).

O álcool é uma droga legalizada e que tem seu uso endossado pela publicidade e incentivado por mensagens explícitas ou subliminares da mídia como uma fonte de obtenção de prazer, o que contribui para o uso abusivo dessa substância por adolescentes, jovens adultos e adultos, podendo acarretar sérios prejuízos biopsicossociais. Isso faz que o consumo disseminado dessa substância seja considerado um problema de saúde pública. As políticas públicas ainda são tímidas frente à magnitude dos agravos gerados e a prevenção é complexa, pois deve levar em conta a subjetividade do sujeito, seus aspectos socioculturais e suas vulnerabilidades específicas, o que requer que se considere cada situação como um caso único. Dessa maneira, um programa consistente de prevenção deve contar com a atuação de uma equipe multiprofissional, com foco em ações psicoprofiláticas, educativas e clínicas e, acima de tudo, com um cuidado humanizado (Déa, Santos, Itakura, \& Olic, 2004; Tostes, Dias, Reis, Silveira, \& Ronzani, 2020).

Conforme Déa et al. (2004), a maioria das pessoas jovens acaba se afastando do uso demasiado do álcool sem receber qualquer tipo de assistência; entretanto, quando o hábito de beber em excesso não é abando- 
nado, os(as) usuários(as) podem se tornar suscetíveis a uma série de consequências nocivas. Logo, é importante que haja um trabalho coordenado de diversos programas intersetoriais direcionados à prevenção e promoção de saúde na passagem dessa etapa do ciclo vital (Scaduto, Barbieri, \& Santos, 2014, 2015). A estratégia de redução de danos (RD) foi adotada no Brasil quando os índices de transmissão do HIV estavam elevados e relacionados ao uso indevido de drogas injetáveis, com compartilhamento de seringas. A partir dessas premissas, a RD pôde ser reformulada e teve seu conceito ampliado - passando a focalizar não somente os usuários de drogas injetáveis -, tornando-se uma medida alternativa à lógica da abstinência.

Assim, a RD passou a ser considerada como um novo paradigma ético-político para a implementação de políticas públicas relacionados ao uso de álcool e/ou outras SPAs, pautadas na cogestão e no cuidado de si, direcionando o(a) usuário(a) para modos de produzir saúde. Dessa maneira, os(as) usuários(as) de SPAs são vistos(as) como responsáveis pela produção de sua saúde, na medida em que tomam para si a tarefa de autocuidado, potencializando as disponibilidades de cuidados dentro de uma esfera democrática e participativa (Passos \& Souza, 2011).

Com relação ao uso de tabaco, percebe-se que, ainda que existam leis restritivas que arrefeceram o consumo, persiste um padrão de uso que chama atenção (Laranjeiras et al., 2012; Organização Mundial da Saúde, 2008). Existe atualmente um corpo robusto de pesquisas e mídias relacionadas ao tabaco e à qualidade de vida - até mesmo a obrigatoriedade de veicular os malefícios do uso em sua própria embalagem de comércio - e tais informações fornecidas à população são consideravelmente eficazes para evidenciar os danos potenciais do uso desse produto à saúde da população (Faustino, Rovinski, Faria, \& Kovelis, 2016; Masood et al., 2015; United Nations Office on Drugs and Crime, 2017). Segundo o relatório da Organização Mundial de Saúde (OMS) divulgado em 2008, o consumo de tabaco é considerado uma epidemia global e está associado a vários agravos à saúde e à sociedade como um todo, com prejuízos econômicos e ambientais. Em uma análise de dados obtidos com 95 estudantes universitários fumantes de Porto Alegre, Brasil, em pesquisa conduzida por Lopes, Cunha, Zibetti e Bizarro (2014), quase $70 \%$ dos fumantes entrevistados relataram que frequentar a faculdade incitava o desejo de fumar. Os autores concluíram que o ambiente universitário pode ser considerado um fator potencial de risco para o tabagismo. Na investigação relacionada ao consumo de tabaco, os dados mostraram que alguns comportamentos evocam uma resposta na maioria dos fumantes universitários: o uso do álcool foi mencionado por quase $96 \%$ dos entrevistados como eliciador do desejo de fumar, seguido de reuniões sociais e situações de estresse, com aproximadamente $95 \%$ das respostas.

Conviver em grupo torna o indivíduo suscetível a se envolver em questões que surgem do convívio social, e o uso abusivo de drogas não é diferente, principalmente no que diz respeito à família, que comumente sofre por manter laço afetivo com o sujeito dependente que se mostra vulnerável a crises de angústia (Medeiros, Maciel, Sousa, Tenório-Souza, \& Dias, 2013). É importante ressaltar a necessidade de que haja melhor compreensão dos modelos moralizantes de representação do uso abusivo de álcool, ou seja, seus possíveis efeitos desagregadores nas relações sociais e a atribuição que frequentemente se dá à dependência como destruidora de unidades sociais essenciais como a família.

Embora o álcool seja frequentemente considerado como facilitador do estabelecimento de relações no âmbito social, para uma parcela da população é um fator que causa rupturas no campo das vivências sociais, acarretando perda de emprego, tensões familiares, relações turbulentas, comportamentos disfuncionais e deterioração dos laços sociais. Entre estudantes, o uso abusivo de álcool tem sido frequentemente associado ao envolvimento em acidentes, brigas, dificuldades no desempenho acadêmico e problemas com a lei (Horta, Horta, \& Horta, 2012).

Por outro lado, as propostas mais recentes na área de saúde pública evidenciam novas expectativas em relação ao papel da família, ressaltando a sua responsabilidade no processo de promoção e prevenção da saúde de seus membros. O campo psi da família vem ganhando visibilidade teórica e relevância no modelo assistencial. A família tem sido vista como sujeito político que luta em busca de direitos, promove cuidados e que deve ser necessariamente incluída nos projetos terapêuticos e nos serviços de saúde, haja vista o que preconiza a Lei de Reforma Psiquiátrica no Brasil - Lei no 10.216/2001 (Brasil, 2001; Rosa, 2005).

É de suma importância qualificar a atuação do profissional de Psicologia nessa área e, mais ainda, é necessário que haja capacitação específica, assim como 
dos demais profissionais de saúde, para uma assistência integral, focando na prevenção e no autocuidado. Deve-se, assim, buscar aprofundar o diálogo com profissionais de outras áreas da saúde, mas não se limitar somente a eles(as), empenhando-se para a formação de equipes multidisciplinares voltadas à promoção de saúde (Déa et al., 2004). Essa é outra razão que torna relevante investigar o consumo de SPAs em estudantes de Psicologia, uma vez que serão os(as) futuros(as) profissionais que poderão atuar nesse campo.

Universitários da área da saúde, e não somente da área de saúde mental, constituem um grupo especial de atenção e preocupação no que se diz respeito ao consumo de SPAs (Botti, Lima, \& Simões, 2010; Pedrosa et al., 2011; Silva et al., 2013; Zeferino et al., 2015). Um dos fatores que incrementam a vulnerabilidade é o fato de estarem envolvidos com a missão de promover saúde e prevenção de diversas morbidades, aproximando-se frequentemente de contextos de sofrimento e dor. Partindo desses pressupostos, discentes das ciências humanas, como do curso de Psicologia, que se voltam para a área da saúde devem ser priorizados(as) pelos programas de prevenção e promoção de saúde direcionados ao consumo de álcool e outras substâncias psicoativas, pois, ao praticarem sua profissão, estarão essencialmente envolvidos na assimilação, prevenção e busca de tratamento de sujeitos cujos problemas são vinculados ao uso de SPAs (Santos et al., 2013).

Pesquisa realizada com estudantes da área de humanas (Silva et al., 2015) mostra que o consumo é maior quando eles(as) são comparados(as) com universitários da área de exatas e biológicas (Andrade et al., 2010), porém, estudos que avaliam especificamente o consumo de SPAs em acadêmicos(as) de Psicologia são escassos (Santos et al., 2013), o que acentua a importância de investigar essa população. Considerando o exposto, este estudo tem como objetivo avaliar o padrão de uso de álcool e outras SPAs - inclusive no padrão binge - e suas possíveis relações com variáveis sociodemográficas em estudantes de Psicologia de uma universidade pública de um município do Triângulo Mineiro, Minas Gerais.

\section{Método}

Trata-se de um estudo descritivo do tipo transversal. Descritivo, porque busca responder se há associação entre o consumo de álcool e outras SPAs e variáveis como sexo, idade, estado civil e classe socioeconômica. O estudo transversal é um tipo de pesquisa observacional que analisa dados coletados ao longo de um período em uma amostra de população predefinida. Mostra uma "fotografia" do momento em que foram coletados os dados e também é conhecido como estudo de prevalência.

\section{Amostra e cenário local}

De um total de 419 estudantes de Psicologia, participaram $180(42,9 \%)$ alunos regulares do curso de graduação de uma universidade pública do estado de Minas Gerais, Brasil. Os critérios de inclusão foram: estar matriculado(a) e presente nos dias da coleta de dados e ter interesse em participar da pesquisa. Os critérios de exclusão foram: não estar na sala de aula no momento da aplicação do instrumento, nem nas duas tentativas posteriores; estar embriagado(a) ou sob efeito de álcool e/ou outras drogas no momento da coleta.

Os(as) participantes eram de ambos os sexos (122 mulheres e 58 homens), com idade variando de 17 a 42 anos. Quanto ao estado civil, a maioria da amostra era solteira. Em relação à classificação econômica, $97,5 \%$ dos(as) participantes eram de classe média.

\section{Instrumentos}

Para a coleta de dados, foi elaborado um questionário composto por informações sociodemográficas (idade, escolaridade, estado civil, entre outras), e questões relacionadas ao consumo de álcool e outras SPAs (Alcohol Use Disorder Identification Test - AUDIT - e Smoking and Substance Involvement Screening Test - ASSIST), possíveis motivos para iniciar-se no consumo (influência de amigos, família, por conta própria). Para avaliar as questões socioeconômicas foi utilizado o Critério de Classificação Econômica Brasil, proposto pela Associação Brasileira de Empresas de Pesquisa - ABEP (2012). As questões investigadas abrangeram a posse de bens e itens de conforto doméstico e o grau de instrução do chefe da família. Cada item possui uma pontuação e a classe econômica é definida pela soma dos escores. $\mathrm{O}$ instrumento foi submetido a um pré-teste com estudantes de outros cursos de graduação e os resultados demonstraram bom entendimento e adequação das questões formuladas.

AUDIT é um instrumento de triagem e acompanhamento do uso problemático do álcool, desenvolvido pela Organização Mundial de Saúde - OMS (Babor, La Fuente, Saunders, \& Grant, 1992). É com- 
posto por dez questões cujas respostas são pontuadas de um a quatro. Esse instrumento foi validado em diversos países, inclusive no Brasil (Mendéz, 1999), com bons níveis de sensibilidade $(87,8 \%)$ e especificidade (81\%) para detecção do uso nocivo de álcool.

ASSIST é um instrumento que avalia o consumo de álcool e de outras SPAs desenvolvido pela World Health Organization - WHO (2002), traduzido e validado para o contexto brasileiro (Henrique, Micheli, Lacerda, Lacerda, \& Formigoni, 2004). Contém oito questões que abrangem nove classes de SPAs (tabaco, maconha, cocaína, álcool, alucinógenos, inalantes, sedativos, opiáceos e estimulantes), que abordam a frequência do uso de determinada(s) substância(s) pelo sujeito no período de três meses e em toda a vida (Formiga, 2013; Henrique et al., 2004). Também são formuladas perguntas que dizem respeito ao consumo de álcool, sua importância na vida e quantidade ingerida, e um possível motivo que teria levado ao início do uso. Os dados de validação do instrumento evidenciaram alpha de Cronbach variando entre as classes de substâncias de 0,80 para álcool, 0,81 para cocaína, 0,79 maconha e 0,80 para tabaco (Henrique et al., 2004).

\section{Procedimento}

A coleta de dados ocorreu entre agosto e outubro de 2016 e foi operacionalizada por meio de aplicação coletiva realizada em salas de aula. Após obtenção da autorização do professor responsável pela sala, a equipe de pesquisa esclarecia os objetivos do estudo, assegurando que a participação dos(as) alunos(as) era voluntária e que seria garantido o anonimato. Aqueles(as) que aceitaram colaborar com a pesquisa assinaram o termo de consentimento livre e esclarecido (TCLE) e foram convidados(as) a preencher os instrumentos. Participantes menores de dezoito anos foram solicitados(as) a assinar o termo de assentimento e seus pais, o TCLE.

O estudo foi aprovado pelo Comitê de Ética em Pesquisa da instituição a qual a equipe de pesquisadoras estava vinculada (CAAE: 42585115.9.0000.5152). Após a assinatura do TCLE, foram distribuídos os instrumentos e sanadas as dúvidas dos(as) participantes. Os questionários respondidos foram colocados pelos(as) estudantes em um envelope lacrado e depositados em uma urna à vista de todos. Os TCLEs assinados foram colocados em outro envelope lacrado.

Os dados foram digitados em uma planilha Excel e validados mediante dupla digitação. Em seguida foram transpostos para o Statistical Package for the Social Sciences (SPSS) versão 21. Os resultados estão apresentados em tabelas com distribuição numérica e de frequência. O teste qui-quadrado foi utilizado para comparar as variáveis. O nível de significância adotado foi p $\leq 0,05$.

\section{Resultados}

A Tabela 1 apresenta as características sociodemográficas dos(as) estudantes de Psicologia e o uso de álcool no padrão binge. Considerando o total de 180 entrevistados, houve predomínio do sexo feminino (67,8\%), jovens com idade entre 20 e 29 anos, a maioria não tinha companheiro, apenas estudavam, viviam com a família e pertenciam à classe média. Os(as) estudantes não se diferenciaram quando eram comparadas as características sociodemográficas e o consumo de álcool no padrão binge. Pode-se observar que, entre os(as) estudantes bebedores(as) no padrão binge, $61,6 \%$ eram do sexo feminino, 64,6\% tinha entre 20 e 29 anos, a maioria estava sem parceiro(a), vivia com os familiares e pertencia à classe média, com diferenças estatisticamente significantes.

Tabela 1

Distribuição da amostra segundo as características sociodemográficas e uso de álcool no padrão binge dos(as) estudantes de Psicologia, Minas Gerais, 2016 ( $\mathrm{N}=180$ ).

\begin{tabular}{|c|c|c|c|c|c|c|c|c|}
\hline & & \multicolumn{6}{|c|}{ Padrão binge-drinking } & \multirow{3}{*}{ Valor de $p$} \\
\hline & & \multicolumn{2}{|c|}{ Total } & \multicolumn{2}{|c|}{ Sim } & \multicolumn{2}{|c|}{ Não } & \\
\hline & & $\mathrm{N}$ & $\%$ & $\mathrm{n}$ & $\%$ & $\mathrm{~N}$ & $\%$ & \\
\hline \multirow[t]{2}{*}{ Sexo } & Masculino & 58 & 32,2 & 38 & 38,4 & 20 & 24,7 & \multirow{2}{*}{$\begin{array}{c}\mathrm{x}^{2}(1)=3,82 \\
p=0,051\end{array}$} \\
\hline & Feminino & 122 & 67,8 & 61 & 61,6 & 61 & 75,3 & \\
\hline \multirow[t]{3}{*}{ Faixa etária } & $17-19$ & 61 & 33,9 & 31 & 31,3 & 30 & 37,0 & \multirow{3}{*}{$\begin{array}{c}\mathrm{x}^{2}(1)=3,087 \\
p=0,214\end{array}$} \\
\hline & $20-29$ & 110 & 61,1 & 64 & 64,6 & 46 & 56,8 & \\
\hline & $>30$ & 9 & 5,0 & 4 & 4,0 & 5 & 5,0 & \\
\hline
\end{tabular}




\begin{tabular}{|c|c|c|c|c|c|c|c|c|}
\hline & & \multicolumn{6}{|c|}{ Padrão binge-drinking } & \multirow{3}{*}{ Valor de $p$} \\
\hline & & \multicolumn{2}{|c|}{ Total } & \multicolumn{2}{|c|}{ Sim } & \multicolumn{2}{|c|}{ Não } & \\
\hline & & $\mathrm{N}$ & $\%$ & $\mathrm{n}$ & $\%$ & $\mathrm{~N}$ & $\%$ & \\
\hline \multirow[t]{2}{*}{ Trabalha } & Sim & 16 & 8,9 & 9 & 9,1 & 7 & 8,6 & \multirow{2}{*}{$\begin{array}{c}\mathrm{x}^{2}(1)=0,11 \\
p=0,916\end{array}$} \\
\hline & Não & 164 & 91,1 & 90 & 90,9 & 74 & 91,4 & \\
\hline \multirow[t]{2}{*}{ Estado civil } & Sem parceiro & 174 & 96,7 & 98 & 99,6 & 76 & 93,8 & \multirow{2}{*}{$\begin{array}{c}\mathrm{x}^{2}(1)=0,07 \\
p=0,932\end{array}$} \\
\hline & Com parceiro & 6 & 3,3 & 1 & 1,0 & 5 & 6,2 & \\
\hline \multirow[t]{3}{*}{ Moradia } & $\begin{array}{l}\text { Amigo/república; } \\
\text { namorado/moradia } \\
\text { estudantil }\end{array}$ & 27 & 15,0 & 20 & 20,2 & 7 & 8,8 & \multirow{3}{*}{$\begin{array}{c}\mathrm{x}^{2}(1)=4,282 \\
p=0,233\end{array}$} \\
\hline & Família & 136 & 75,6 & 68 & 68,7 & 68 & 85,0 & \\
\hline & Sozinho/outros & 16 & 8,9 & 11 & 11,1 & 5 & 6,3 & \\
\hline \multirow[t]{3}{*}{ Classe social } & Alta & 13 & 7,2 & 11 & 11,1 & 2 & 2,5 & \multirow{3}{*}{$\begin{array}{c}\mathrm{x}^{2}(1)=7,600 \\
p=0,022\end{array}$} \\
\hline & Média & 164 & 90,6 & 86 & 85,9 & 78 & 97,5 & \\
\hline & Baixa & 3 & 1,7 & 3 & 3,0 & - & - & \\
\hline
\end{tabular}

Valor de $p \leq 0,05$

A prevalência do uso de álcool entre estudantes de Psicologia foi de $81,7 \%$ na vida, $67,6 \%$ nos últimos três meses e $55 \%$ no padrão binge drink (dados não apresentados em tabelas). Outro aspecto apontado na Tabela 2 é que mais da metade já consumiu álcool no padrão binge, $27,2 \%$ raramente e $14,4 \%$ ocasionalmente.

Os principais fatores motivadores alegados pelos(as) estudantes para experimentarem bebidas alcoólicas ou iniciarem o seu consumo foram: iniciativa própria $(39,9 \%)$, por incentivo dos amigos $(17,1 \%)$ e por incentivo da família $(8,7 \%)$. Esses dados não foram apresentados em tabela.

$\mathrm{Na}$ amostra total, o uso de álcool se destacou dentre as substâncias mais utilizadas por estudantes de Psicologia $(81,7 \%)$, seguido de maconha $(46,1 \%)$ e tabaco (45,6\%). As substâncias psicoativas associadas ao uso de álcool mais consumidas na vida pelos(as) estudantes de Psicologia, nos últimos três meses e no padrão binge, foram maconha, tabaco, alucinógenos e os hipnóticos sedativos, dados apresentados na Tabela 3.

Tabela 2

Frequência de consumo do álcool no padrão binge na amostra de estudantes de Psicologia, Minas Gerais, $2016(\mathrm{~N}=180)$.

\begin{tabular}{lc}
\hline \multicolumn{1}{c}{ Consumo no padrão binge } & $\mathrm{N}(\%)$ \\
\hline Nunca & $81(45)$ \\
Menos que uma vez por mês & $49(27,2)$ \\
Mensalmente & $26(14,4)$ \\
Semanalmente & $23(12,8)$ \\
Diariamente & $1(0,6)$ \\
\hline
\end{tabular}

\section{Tabela 3}

Associação entre uso de álcool e outras substâncias psicoativas na vida, nos últimos três meses e no padrão binge, entre estudantes de Psicologia, Minas Gerais, 2016 ( $\mathrm{N}=180$ ).

\begin{tabular}{|c|c|c|c|c|c|c|}
\hline & \multicolumn{6}{|c|}{ Consumo de álcool } \\
\hline & \multicolumn{2}{|c|}{ Na vida } & \multicolumn{2}{|c|}{ Nos últimos 3 meses } & \multicolumn{2}{|c|}{ Binge-drinking } \\
\hline & $\mathrm{N}$ & $\%$ & $\mathrm{~N}$ & $\%$ & $\mathrm{~N}$ & $\%$ \\
\hline Maconha & 69 & 47,3 & 64 & $53,8^{*}$ & 61 & $61,6^{*}$ \\
\hline Tabaco & 19 & $13,0^{*}$ & 67 & $56,3^{*}$ & 68 & $68,7^{*}$ \\
\hline Alucinógenos & 19 & $13,0^{*}$ & 18 & $15,1^{*}$ & 16 & $16,3^{*}$ \\
\hline Hipnóticos/sedativos & 19 & 10,6 & 14 & 12,0 & 12 & 12,5 \\
\hline Anfetaminas e êxtase & 14 & 9,7 & 13 & 11,1 & 12 & $12,5^{*}$ \\
\hline Inalantes & 13 & 9,0 & 11 & 9,4 & 12 & $12,5^{*}$ \\
\hline Cocaína/crack & 13 & 9,0 & 11 & 11,4 & 9 & 9,4 \\
\hline
\end{tabular}




\section{Discussão}

O consumo de álcool e de outras SPAs tem aumentado entre o público universitário nos últimos anos (Santos et al., 2013; Soares et al., 2014). Neste estudo, buscou-se compreender esse fenômeno a partir de uma avaliação delineada para caracterizar o consumo de álcool e de outras SPAs, de modo a sistematizar informações para subsidiar programas de prevenção voltados às necessidades e particularidades dessa população.

Em relação ao sexo, a amostra era composta majoritariamente por mulheres, o que é congruente com a distribuição encontrada em outros estudos (Silva et al., 2015; Soares et al., 2014). Dentre os respondentes, notou-se que o consumo feminino $(67,8 \%)$ era maior do que o masculino (32,2\%). Esse aumento no consumo feminino já havia sido detectado anteriormente por Silva et al. (2006) em um estudo com 926 universitários da área de ciências biológicas de uma instituição e nível superior de São Paulo, no qual se constatou um consumo de álcool na vida para os homens da ordem de $85,4 \%$ e, para as mulheres, de $84,5 \%$.

Ao ingressar no ensino superior, o processo de assumir novas responsabilidades faz com que o(a) jovem fique mais exposto(a) a situações de contato com diversas SPAs, mostrando-se mais suscetível à propaganda e aos apelos da mídia e do ambiente estudantil permissivo, que apoia e divulga maciçamente os supostos prazeres relacionados ao uso de álcool. Ademais, o álcool é uma substância presente na vida dos jovens desde a infância até a idade adulta. Entretanto, estudos indicam que jovens adultos(as) e solteiros(as) ingerem mais álcool do que adultos(as) com parceiros(as) (Petroianu, Reis, Cunha, \& Souza, 2010; Zeferino et al., 2015). Esses achados são consistentes com os resultados obtidos nesta pesquisa (Tabela 1).

Outro aspecto do presente estudo que é consistente com achados de outras investigações científicas foi a caracterização da amostra, como visto na Tabela 1 , composta por maioria $(64,6 \%)$ de universitários(as) com idade entre 20 e 29 anos, sem parceiros(as) fixos(as) (96,7\%) e pertencentes à classe média (90,6\%). Soares et al. (2014) chamam a atenção para algumas características dessa população, como o fato de ter mais tempo livre e desfrutar de uma boa renda familiar, com maior autonomia concedida pelos pais.

Para Malta et al. (2014), os danos causados pelo consumo de álcool por adolescentes são diferentes dos observados em adultos e o padrão de uso é mais episódico. O álcool é uma droga que não é consumida com tanta frequência como se observa em adultos, porém, segundo a Pesquisa Nacional de Saúde Escolar - PeNSE (Malta et al., 2012), o consumo de álcool aumenta conforme a idade. Dos(as) estudantes que participaram da pesquisa, menos do que a metade não consumia álcool no padrão binge-drinking, entretanto, a outra metade referiu beber em binge, como mostra a Tabela 2 (Laranjeira et al., 2012).

No presente estudo, o álcool foi associado ao uso do tabaco nos últimos três meses e no padrão binge-drinking (Tabela 3). O tabaco foi a substância mais consumida pelos estudantes de Psicologia na presença do álcool. Esses dados devem ser analisados juntamente com os motivos alegados para o uso, notadamente a questão do convívio universitário e da simples presença no âmbito da universidade como favorecedora do consumo.

Segundo pesquisa realizada por Lopes et al. (2014), são vários os motivos que levam os estudantes a fumar e um deles é o convívio na universidade. Porém, o motivo mencionado por quase todos os entrevistados(as) foi que o álcool atua como potencializador do desejo de fumar, seguido do contexto de festas/reuniões sociais e da busca de alívio de situações de estresse. Por que o álcool seria tão determinante no desencadeamento do desejo de fumar? Esse é um fenômeno bastante comum e já conhecido da literatura: estudo realizado com 322 alunos de Medicina da Universidade Federal de Minas Gerais mostrou que os(as) estudantes que consumiam bebidas alcoólicas estavam mais propensos ( 1,23 vezes) ao consumo de cigarro (Petroianu et al., 2010). Estudo realizado com 250 graduandos(as) de universidades de Santa Catarina, Caribe e América Latina mostrou que o álcool e o tabaco estão muito presentes em festas, logo, a facilidade de acesso e a necessidade de se sentir pertencente ao grupo podem levar a um aumento do consumo (Zeferino et al., 2015).

Como o álcool é uma substância lícita e socialmente tolerada, e que pode ser facilmente encontrada e adquirida sem muitas restrições no país, torna-se uma SPA que tem amplo poder de penetração e alcance na sociedade. A maioria da população está familiarizada com as marcas comerciais, logotipos e propriedades devido à intensa comercialização e publicidade da substância. O presente estudo contribui para o avanço do conhecimento na área ao evi- 
denciar que o álcool também é a substância psicoativa que os(as) estudantes de Psicologia mais consumiram ao longo da vida no padrão binge e nos últimos três meses, como mostra a Tabela 3.

Esse resultado é consistente com os achados obtidos pelo levantamento da Senad, que também encontrou que o álcool era a substância mais consumida pelos(as) jovens, seguido do tabaco e da maconha. Ao examinar os dados do presente estudo, nota-se que a maconha foi apontada como a substância psicoativa ilícita mais consumida ao longo da vida dos(as) estudantes de Psicologia, seguida de alucinógenos e tabaco, aliada ao consumo de álcool (Tabela 3). Nos últimos três meses, as substâncias que os estudantes fizeram mais uso em conjunto com o álcool foram o tabaco e a maconha. A investigação do padrão binge-drinking também mostrou que essas substâncias são as mais consumidas por estudantes de Psicologia. Desse modo, álcool, tabaco e maconha foram as drogas mais consumidas. É fato que, sob efeito do álcool - uma droga de mais fácil acesso e comercialização - as pessoas tendem a "aproveitar o embalo" e fumar e/ou ingerir outras substâncias, de modo que a bebida alcoólica funciona como potencializador do uso de outras drogas.

Além de conhecer as substâncias mais consumidas, identificar os padrões de uso pela população é de extrema importância para o planejamento de ações e intervenção precoce, anteriores a um uso problemático ou dependente do álcool (Reis, Machado, Andrade, \& Figueiredo, 2017). Déa et al. (2004) complementam esse dado lembrando que essas intervenções devem ser articuladas com programas de prevenção e estratégias de promoção de saúde voltadas para essa etapa da vida, caracterizada por uma transição psicossocial. Também é fundamental favorecer condições para uma efetiva presença da família na vida escolar de adolescentes e jovens, uma vez que se trata de um problema de saúde pública com elevado custo social. A família exerce papel primordial na construção de uma nova trajetória de vida para o(a) familiar que se encontra em uma fase de ajustamento à universidade, devendo ser também parceira e corresponsável pelo cuidado se e quando a pessoa estiver em sofrimento. Mas é importante destacar que a desejável participação da família na vida do(a) jovem universitário(a) é um processo a ser construído, com limites e potencialidades que variam de acordo com cada contexto.

\section{Conclusão}

Os resultados deste estudo evidenciaram que o álcool é a droga mais consumida no meio universitário por estudantes de Psicologia. Os dados apresentados trazem importantes contribuições para a área do conhecimento porque oferecem subsídios para o planejamento de programas de intervenção e prevenção no meio universitário. O conhecimento produzido por esta pesquisa também é inovador, porque até então não se tinham informações específicas sobre o padrão de uso de SPAs entre acadêmicos de Psicologia no país. Conhecer o perfil de consumo e as razões apontadas pelos jovens universitários para o comportamento de usar drogas lícitas e ilícitas são dimensões relevantes para se conhecer a vulnerabilidade desse segmento, especialmente porque se tratam de futuros(as) profissionais de saúde mental que, no exercício da profissão, vão lidar com indivíduos com problemas relacionados ao uso de substâncias.

Por outro lado, não se pode incorrer no equívoco de considerar que o uso intenso de SPAs seja nocivo por si só. Temos de analisar com cuidado os possíveis desdobramentos desse uso no cotidiano dos(as) participantes, examinando principalmente as eventuais interferências no comportamento - envolvimento em acidentes, brigas, problemas com a lei - e em funções relacionadas aos estudos, como memória, raciocínio, atenção e concentração, levando a problemas de desempenho escolar e dificuldades de ajustamento ao meio acadêmico.

As repercussões que o consumo de álcool e outras SPAs podem ter na vida dos(as) jovens é considerável. Quando ingressam na universidade, os(as) estudantes muitas vezes ficam expostos a situações de vulnerabilidade porque são imaturos(as) e vivenciam um período crítico de transição psicossocial, no qual se tornam mais independentes da supervisão parental e tomam mais decisões a respeito de suas próprias vidas. Frequentemente isso desperta insegurança e instabilidade, deixando os(as) jovens universitários(as) mais suscetíveis à influência dos pares e à busca de diversão e descontração em ambientes nos quais o beber é estimulado e facilitado, assim como o acesso a outros tipos de drogas. Nas "baladas" e festas universitárias, principalmente com open bar, o beber em quantidade excessiva (binge-drinking) pode se associar a outros comportamentos de risco, como dirigir sob efeito do álcool, manter relações sexuais sem proteção ou envolver-se em distúrbios da ordem pública e infrações à lei. 
O álcool aparece ainda como agente potencializador do desejo de fumar e também é visto como fator gregário em festas e reuniões sociais. $\mathrm{O}$ (a) jovem, devido à necessidade de se sentir pertencente ao grupo, busca esses ambientes para se socializar e, muitas vezes, procura o álcool como facilitador do enfrentamento de situações de estresse relacionadas às pressões da vida universitária.

A partir do conhecimento obtido no presente estudo, de que estudantes do curso de Psicologia fazem uso abusivo de SPAs e não contam com suporte social e comunitário adequados, é relevante que haja um esforço redobrado destinado à implementação de programas de prevenção e políticas públicas que regulem o consumo de álcool e/ou de outras SPAs no cenário universitário. Esses programas devem ser guiados por evidências científicas e necessitam gravitar em direção ao fortalecimento do cuidado de si próprio, empoderando os(as) jovens estudantes como protagonistas da própria história e como sujeitos que participam ativamente de suas decisões e comportamentos de busca de saúde.

É necessário investir na oferta de alternativas de sociabilidade juvenil - grupos de convivência, clubes e espaços universitários voltados à socialização - onde estudantes possam ter acesso à busca de fruição do prazer, beleza e popularidade. Assim como também é preciso qualificar o debate nacional, hoje bastante empobrecido, sobre a possível descriminalização das drogas, considerando a necessidade de buscar um novo paradigma ético-político para a implementação de políticas públicas relacionadas ao uso de álcool e/ ou de outras SPAs, pautadas na cogestão e no cuidado de si. É preciso ter em mente que novos horizontes são possíveis e que é possível direcionar o(a) usuário(a) de SPAs para a busca de modos protetivos de produzir saúde, potencializando cuidados dentro de um espírito participativo e democrático.

Este estudo possibilitou uma análise do uso nocivo de substâncias psicoativas por estudantes de Psicologia de uma universidade pública, buscando conhecer os comportamentos de consumo abusivo. Constatou-se o quão onipresente é o álcool e outras SPAs no contexto universitário, corroborando resultados obtidos por outras pesquisas que indicam que se trata de um fenômeno multideterminado, inscrito na cultura e no comportamento de jovens, mas cujas raízes remontam à aprendizagem social de hábitos adquiridos desde a infância.
É necessária uma atenção diferenciada principalmente às drogas socialmente toleradas e legalizadas, como álcool e tabaco, que são insufladas por um comércio próspero e lucrativo que as vende como passaporte para se usufruir de uma vida social mais descontraída, potente e glamourosa. Por outro lado, as drogas ilícitas carregam o estigma da criminalização. É provável que, ao chegar à universidade, a aprendizagem social que teve início na infância e adolescência retorne devido ao ambiente estressante, como apontado, e a quantidade de festas, reuniões e comemorações regadas a diferentes substâncias.

Em contrapartida, os dados obtidos sugerem o quão recente é o início do consumo de álcool pela maioria dos(as) estudantes, além de também indicar que álcool e tabaco foram as drogas mais utilizadas por essa população, assim como a maconha, sendo esta do campo das drogas cujo uso é marginalizado e criminalizado no país. Os resultados reverberam as pesquisas que sugerem que há um número crescente de jovens que fazem uso de álcool e outras SPAs. Desse modo, o estudo permite inferir, ainda que indiretamente, o potencial impacto do álcool e de outras SPAs na vida das pessoas e de suas famílias, além de elencar alguns dos fatores que desencadeiam o uso abusivo dessas substâncias.

Ao depararmos com o consumo problemático de álcool e outras SPAs, percebemos a importância da criação de políticas preventivas para equacionar essa situação que, na verdade, está ancorada em um problema social mais amplo. Nesse sentido, ações preventivas devem ser iniciadas no âmbito das próprias famílias para que possam ser complementadas pelo trabalho educativo promovido pelas escolas em todos os níveis educacionais. é preciso que essas ações sejam continuadas e voltadas para a desmistificação das questões relacionadas ao uso de substâncias. Também é necessário que o uso de SPAs seja visto como uma questão de saúde pública, e não como um problema de ordem moral, legal ou policial, a ser resolvido apenas com políticas repressivas que buscam criminalizar e segregar os(as) usuários(as) do convívio social. As pessoas que já estão em sofrimento existencial devido ao uso problemático de SPAs devem ter acesso a uma assistência humanizada, que potencialize o seu autocuidado e que seja pautado no acolhimento de outras questões que vão além do tratamento dos sintomas e da eventual dependência. 


\section{Referências}

Andrade, A., Duarte, P., \& Oliveira, L. (2010). I Levantamento Nacional sobre o uso de álcool, tabaco e outras drogas entre universitários das 27 capitais brasileiras. Brasília, DF: Senad. https://goo.gl/XMoVz7

Arantes, L. F. R. (2012). Binge drinking: um estudo bibliométrico (1999-2010) dos artigos publicados na base de dados SciELO. Estudos de Psicologia (Campinas), 29(2), 253-257. http://www.scielo.br/pdf/estpsi/v29n2/a11v29n2.pdf

Associação Brasileira de Empresas de Pesquisa. (2012). Critério de classificação econômica Brasil. São Paulo, SP. http://www.abep.org/novo/Content.aspex?ContentID=301

Babor, T. F., La Fuente, J. R., Saunders, J., \& Grant, M. (1992). Audit: The alcohol use disorders identificacion test: guidelines for use in primary healthcare. World Health Organization, 4, 1-29. http://apps.who.int/iris/ bitstream/10665/67205/1/WHO_MSD_MSB_01.6a.pdf

Botti, N. C. L., Lima, A. F. D., \& Simões, W. M. B. (2010). Uso de substâncias psicoativas entre acadêmicos de enfermagem da Universidade Católica de Minas Gerais. SMAD: Revista Eletrônica Saúde Mental Álcool e Drogas, 6(1), 1-16. http://pepsic.bvsalud.org/scielo.php?script=sci_arttext\&pid=S1806-69762010000100013\&lng=pt\&tlng=pt

Brasil. (2001). Lei no 10.216, de 6 de abril de 2001. Dispõe sobre a proteção e os direitos das pessoas portadoras de transtornos mentais e redireciona o modelo assistencial em saúde mental. Brasília, DF. http://www.planalto.gov.br/ ccivil_03/leis/leis_2001/l10216.htm

Déa, H. R. F. D., Santos, E. N., Itakura, E., \& Olic, T. B. (2004). A inserção do psicólogo no trabalho de prevenção ao abuso de álcool e outras drogas. Psicologia: Ciência e Profissão, 24(1), 108-115. https://dx.doi.org/10.1590/ S1414-98932004000100012

Faustino, E. C., Rovinski, E., Faria, F., \& Kovelis, D. (2016). Tabagismo e atividade física: revisão da literatura. Vitrine Produção Acadêmica, 4(2), 21-30. http://www.vitrineacademica.dombosco.sebsa.com.br/index.php/vitrine/ article/viewFile/197/198

Formiga, N. (2013). O consumo de álcool em universitários: Fidedignidade e sensibilidade de uma escala de medida. Estudos Interdisciplinares em Psicologia, 4(2), 130-147. http://pepsic.bvsalud.org/scielo.php?script=sci arttext\&pid=S2236-64072013000200002\&lng=pt\&tlng=pt

Gomes, B. R., \& Capponi, M. (2011). Álcool e outras drogas: novos olhares, outras percepções. In: Conselho Regional de Psicologia (Org.), Álcool e outras drogas. São Paulo, SP: Conselho Regional de Psicologia da 6a Região. http://www.crpsp.org.br/portal/comunicacao/livro-alcool-drogas/crpsp-alcool-e-outras-drogas.pdf

Henrique, I. F. S., Micheli, D., Lacerda, R. B., Lacerda, L. A., \& Formigoni, M. L. O. S. (2004).Validation of the Brazilian version of Alcohol, Smoking and Substance Involvement Screening Test (ASSIST). Revista da Associação Médica Brasileira, 50(2), 199-206. http://www.scielo.br/pdf/ramb/v50n2/20784

Horta, R. L., Horta, B. L., \& Horta, C. L. (2012). Uso de drogas e sofrimento psíquico numa universidade do Sul do Brasil. Psicologia em Revista, 18(2), 264-276. https://dx.doi.org/http://dx.doi.org/10.5752/P.1678-9563.2012v18n2p264

Laranjeira, R., Madruga, C. S., Pinsky, I, Caetano, R., \& Mitsuhiro, S. S. (Orgs.). (2012). Segundo Levantamento Nacional de Álcool e Drogas (Lenad). São Paulo, SP: Inpad. http://inpad.org.br/wp-content/uploads/2014/03/ Lenad-II-Relat\%C3\%B3rio.pdf

Lopes, F., Cunha, S. M., Zibetti, M., \& Bizarro, L. (2014). Padrão de consumo e expectativas em relação ao cigarro entre universitários. Psicologia, Saúde \& Doenças, 15(2), 439-453. http://www.scielo.mec.pt/scielo.php? script=sci_arttext\&pid=S1645-00862014000200009\&lng=pt\&tlng=pt.

Malta, D. C., Machado, I. E., Porto, D. L., Silva, M. M. A, Freitas, P. C., Costa, A. W. N., \& Oliveira-Campos, M. (2014). Consumo de álcool entre adolescentes brasileiros segundo a Pesquisa Nacional de Saúde Escolar (PeNSE 2012). Revista Brasileira de Epidemiologia, 17(1), 203-214. http://dx.doi.org/10.1590/1809-4503201400050016

Masood, S., Cappelli, C., Li, Y., Tanenbaum, H., Chou, C. P., Spruijt-Metz, D., Palmer, P. H., Johnson, A., \& Xie, B. (2015). Cigarette smoking is associated with unhealthy patterns of food consumption, physical activity, sleep impairment, and alcohol drinking in Chinese male adults. International Journal of Public Health, 60(8), 891-899.

Marques, A. C. P. R., \& Cruz, M. S. (2000). O adolescente e o uso de drogas. Revista Brasileira de Psiquiatria, 22(2), 32-36. http://dx.doi.org/10.1590/S1516-44462000000600009 
Medeiros, K. T., Maciel, S. C., Sousa, P. F., Tenório-Souza, F. M., \& Dias, C. C. V. (2013). Representações sociais do uso e abuso de drogas entre familiares de usuários. Psicologia em Estudo (Maringá), 18(2), 269-279. http://dx.doi.org/ $10.1590 /$ S1413-73722013000200008

Mendéz, E. B. (1999). Uma versão brasileira do AUDIT (Alcohol Use Disorders Identification Test) [Dissertação de mestrado]. Universidade Federal de Pelotas, Pelotas, RS.

National Institute on Alcohol Abuse and Alcoholism. (2016). Dietary guidelines for Americans 2015-2020, US Department of Health and Human Services and US Department of Agriculture. http://www.niaaa.nih.gov/ alcohol-health/overview-alcohol-consumption/moderate-binge-drinking

Nimtz, M. A., Tavares, A. M. F., Maftum, M. A., Ferreira, A. C. Z., Borba, L. O., \& Capistrano, F. C. (2014). Impacto do uso de drogas nos relacionamentos familiares de dependentes químicos. Cogitare Enfermagem, 19(4), 667-672. http://revistas.ufpr.br/cogitare/article/view/35721/23905

Organização Mundial da Saúde. (2008). Cuidados de saúde primários: agora mais que nunca. http://www.who.int/ eportuguese/publications/whr08_pr.pdf?ua=1

Passos, E. H., \& Souza, T. (2011). Redução de danos e saúde pública: construções alternativas à política global de "guerra às drogas". Psicologia \& Sociedade, 23(1), 154-162. http://www.scielo.br/pdf/psoc/v23n1/a17v23n1.pdf

Pedrosa, A. A. S., Camacho, L. A. B., Passos, S. R. L., \& Oliveira, R. V. C. (2011). Consumo de álcool entre estudantes universitários. Cadernos de Saúde Pública, 27(8), 1611-1621. https://dx.doi.org/10.1590/S0102-311X2011000800016

Petroianu, A., Reis, D. C., Cunha, B. D., \& Souza, D. M. (2010). Prevalence of alcohol, tobacco and psychotropic drug use among medical students at the Universidade Federal de Minas Gerais. Revista da Associação Médica Brasileira, 56(5), 568-571. http://dx.doi.org/10.1590/S0104-42302010000500019

Reis, F. G., Machado, E. C., Andrade, M., \& Figueiredo, G. L. A. (2017). Conhecimento e abordagens acerca do uso problemático do álcool. Psicologia: Ciência e Profissão, 37(2), 335-348. https://dx.doi.org/10.1590/ 1982-3703000852016

Rosa, L. C. (2005). A inclusão da família nos projetos terapêuticos dos serviços de saúde mental. Psicologia em Revista, 11(18), 205-218. http://pepsic.bvsalud.org/pdf/per/v1ln18/v1ln18a05.pdf

Santos, M. V. F., Pereira, D. S., \& Siqueira, M. M. (2013). Uso de álcool e tabaco entre estudantes de Psicologia da Universidade Federal do Espírito Santo. Jornal Brasileiro de Psiquiatria, 62(1), 22-30. http://dx.doi.org/10.1590/ S0047-20852013000100004

Scaduto, A. A., Barbieri, V., \& Santos, M. A. (2014). Comunidades terapêuticas para dependentes de substâncias psicoativas: avaliação dos resultados do tratamento. Revista Psicologia: Teoria e Prática, 16(2), 156-171. http://dx.doi.org/10.15348/1980-6906/psicologia.v16n2p156171

Scaduto, A. A., Barbieri, V., \& Santos, M. A. (2015). Adesão aos princípios da comunidade terapêutica e processo de mudança ao longo do tratamento. Psicologia: Ciência e Profissão, 35(3), 781-796. https://doi.org/10.1590/ 1982-3703000792013

Silva, E. R., Ferreira, A. C. Z., Borba, L. O., Kalinke, L. P., Nimtz, M. A., \& Maftum, M. A. (2016). Impacto das drogas na saúde física e mental de dependentes químicos. Ciência, Cuidado \& Saúde, 15(1), 101-108. http://dx.doi.org/ 10.4025/cienccuidsaude.v15il.27137

Silva, J. N., Rodrigues, M. G., Kimberly, M. J., Finelli, L. A. C., \& Soares, W. D. (2015). Consumo de álcool entre universitários. Revista Brasileira de Pesquisa em Ciências da Saúde, 2(2), 35-40. http://www.icesp.br/revistas-eletronicas/ index.php/RBPeCS/article/view/45

Silva, L. V. E. R., Malbergier, A., Stempliuk, V. A., \& Andrade, A. G. (2006). Fatores associados ao consumo de álcool e drogas entre estudantes universitários. Revista de Saúde Pública, 40(2), 280-288. https://dx.doi.org/10.1590/ S0034-89102006000200014

Silva, R. P., Souza, P., Nogueira, D. A., Moreira, D. S., \& Chaves, E. C. L. (2013). Relação entre bem-estar espiritual, características sociodemográficas e consumo de álcool e outras drogas por estudantes. Jornal Brasileiro de Psiquiatria, 62(3), 191-198. http://dx.doi.org/10.1590/S0047-20852013000300003

Soares, W. D., Barros, K. S. J., Araújo, T. P., Finelli, L. A. C., \& Jones, K. M. (2014). Álcool como mediador social em universitários. Revista Brasileira de Promoção de Saúde, 28(3), 427-433. http://www.redalyc.org/pdf/408/40844684016.pdf 
Tostes, J. G. A., Dias, R. T., Reis, A. A. S., Silveira, P. S., \& Ronzani, T. M. (2020). Interventions to reduce stigma related to people who use drugs: systematic review. Paidéia (Ribeirão Preto), 30, e3022.

United Nations Office on Drugs andCrime. (2017). WorldDrugReport2017.Vienna:UNODC.https://www.unodc.org/ wdr2017/field/Booklet_1_EXSUM.pdf

Vilela, T. R., Silva, R. S., Grandi, C. G., Rocha, M. M., \& Figlie, N. B. (2016). Emotional and behavioral problems in children living with addicted family members: Prevention challenges in an underprivileged suburban community. Paidéia (Ribeirão Preto), 26(64), 225-234.

WHO Assist Working Group. (2002). The Alcohol, Smoking and Substance Involvement Screening Test (ASSIST): development, reliabilityand feasibility. Addiction, 1183-1194.http:// dx.doi.org/10.1046/j.1360-0443.2002.00185.x

Zeferino, M. T., Hamilton, H., Brands, B., Wright, M. da G. M., Cumsille, F., \& Khenti, A. (2015). Drug consuption among university students: Family, spirituality and entertainment moderating influence of pairs. Texto \& Contexto Enfermagem, 24(spe), 125-135. http://dx.doi.org/10.1590/0104-07072015001150014

\section{Isabella Tereza Martins Pires}

Psicóloga pelo Instituto de Psicologia da Universidade Federal de Uberlândia, Uberlândia - MG. Brasil.

E-mail: isabellatmp1@gmail.com

(1) https://orcid.org/0000-0002-0213-7348

\section{Marciana Gonçalves Farinha}

Professora adjunta do Instituto de Psicologia da Universidade Federal de Uberlândia, Uberlândia - MG. Brasil.

E-mail:marciana@ufu.br

(1) https://orcid.org/0000-0002-2024-7727

\section{Sandra Cristina Pillon}

Professora titular do Programa de Pós-Graduação em Enfermagem Psiquiátrica da Escola de Enfermagem de Ribeirão Preto da Universidade de São Paulo, Ribeirão Preto - SP. Brasil.

E-mail pillon@eerp.usp.br

(1) https://orcid.org/0000-0001-8902-7549

\section{Manoel Antônio dos Santos}

Professor titular do Programa de Pós-Graduação em Psicologia da Faculdade de Filosofia, Ciências e Letras de Ribeirão Preto da Universidade de São Paulo, Ribeirão Preto - SP. Brasil.

E-mail: masantos@ffclrp.usp.br

(1) https://orcid.org/0000-0001-8214-7767

Endereço para envio de correspondência: Instituto de Psicologia da Universidade Federal de Uberlândia, Campus Umuarama, Bloco 2C, Sala 40/43. Av. Pará, 1720, Umuarama. CEP: 38400-902. Uberlândia - MG. Brasil.

Recebido 16/02/2018

Aceito 06/02/2019

Received 16/02/2018

Approved 06/02/2019

Recibido 16/02/2018

Aceptado 06/02/2019 
Psicologia: Ciência e Profissão 2020 v. 40, e191670, 1-14.

Como citar: Pires, I. T. M., Farinha, M. G., Pillon, S C., \& Santos, M. A. (2020). Uso de Álcool e outras Substâncias Psicoativas por Estudantes Universitários de Psicologia. Psicologia: Ciência e Profissão, 40, 1-14.

https://doi.org/10.1590/1982-3703003191670

How to cite: Pires, I. T. M., Farinha, M. G., Pillon, S C., \& Santos, M. A. (2020). Use of Alcohol and Other Psychoactive Substances by University Students of Psychology. Psicologia: Ciência e Profissão, 40, 1-14. https://doi.org/10.1590/1982-3703003191670

Cómo citar: Pires, I. T. M., Farinha, M. G., Pillon, S C., \& Santos, M. A. (2020). Uso de Alcohol y otras Sustancias Psicoactivas por Estudiantes Universitarios de Psicología. Psicologia: Ciência e Profissão, 40, 1-14.

https://doi.org/10.1590/1982-3703003191670 